\title{
Gypenosides regulate farnesoid $X$ receptor- mediated bile acid and lipid metabolism in a mouse model of non-alcoholic steatohepatitis
}

Hongshan $\mathrm{Li}^{1,2,3^{*}}$, Yingfei $\mathrm{Xi}^{4}$, Xin Xin ${ }^{1}$, Huajie Tian ${ }^{1}$ and Yiyang $\mathrm{Hu}^{{ }^{*}}$

\begin{abstract}
Background: Gypenosides (Gyp) are the main ingredient of the Chinese medicine, Gynostemma pentaphyllum. They are widely used in Asia as a hepatoprotective agent. Here, we elucidated the mechanism of Gyp in non-alcoholic steatohepatitis (NASH) with a focus on farnesoid X receptor (FXR)-mediated bile acid and lipid metabolic pathways.

Methods: NASH was induced in mice by high-fat diet (HFD) feeding, while mice in the control group were given a normal diet. At the end of week 10, HFD-fed mice were randomly divided into HFD, HFD plus Gyp, and HFD plus obeticholic acid (OCA, FXR agonist) groups and were given the corresponding treatments for 4 weeks. Next, we analyzed the histopathological changes as well as the liver triglyceride (TG) level and serum alanine aminotransferase (ALT), aspartate aminotransferase (AST), fasting blood glucose (FBG), fasting insulin (FINS), TG, total cholesterol (TC), and low-density lipoprotein cholesterol (LDL-C) levels as well as the bile acid profile. We carried out RT-PCR and western blotting to detect HFD-induced alterations in gene/protein expression related to bile acid and lipid metabolism.

Results: The HFD group had histopathological signs of hepatic steatosis and vacuolar degeneration. The liver TG and serum ALT, AST, FBG, FINS, TC, and LDL-C levels as well as the total bile acid level were significantly higher in the HFD group than in the control group $(P<0.01)$. In addition, we observed significant changes in the expression of proteins involved in bile acid or lipid metabolism $(P<0.05)$. Upon treatment with Gyp or OCA, signs of hepatic steatosis and alterations in different biochemical parameters were significantly improved $(P<0.05)$. Further, HFDinduced alterations in the expression genes involved in bile acid and lipid metabolism, such as CYP7A1, BSEP, SREBP1, and FASN, were significantly alleviated.
\end{abstract}

Conclusions: Gyp can improve liver lipid and bile acid metabolism in a mouse model of NASH, and these effects may be related to activation of the FXR signaling pathway.

Keywords: Gypenosides, Farnesoid X receptor, Bile acid, Lipid metabolism

\footnotetext{
*Correspondence: Lihongshan_1982@126.com; yyhuliver@163.com

${ }^{1}$ Institute of Liver Disease, Shuguang Hospital, Shanghai University of Traditional Chinese Medicine, Shanghai, China

Full list of author information is available at the end of the article
}

C C The Author(s). 2020 Open Access This article is licensed under a Creative Commons Attribution 4.0 International License, which permits use, sharing, adaptation, distribution and reproduction in any medium or format, as long as you give appropriate credit to the original author(s) and the source, provide a link to the Creative Commons licence, and indicate if changes were made. The images or other third party material in this article are included in the article's Creative Commons licence, unless indicated otherwise in a credit line to the material. If material is not included in the article's Creative Commons licence and your intended use is not permitted by statutory regulation or exceeds the permitted use, you will need to obtain permission directly from the copyright holder. To view a copy of this licence, visit http://creativecommons.org/licenses/by/4.0/ The Creative Commons Public Domain Dedication waiver (http://creativecommons.org/publicdomain/zero/1.0/) applies to the data made available in this article, unless otherwise stated in a credit line to the data. 


\section{Background}

Non-alcoholic fatty liver disease (NAFLD) is a complication of obesity and metabolic syndromes [1]. The clinical presentation of NAFLD ranges from simple steatosis and non-alcoholic fatty liver to hepatic fibrosis [2]. Hepatic fibrosis and non-alcoholic steatohepatitis (NASH) are among the main reasons for the development of liver cirrhosis and hepatocellular carcinoma [3, 4].

Along with increased obesity rates, the global prevalence of NAFLD has reached up to $24 \%$, creating a huge economic burden on healthcare systems worldwide [5]. Therefore, implementing strategies that lead to the prevention and treatment of NASH and NAFLD will have a significant socioeconomical impact. To date, dietary adjustments and lifestyle changes are the standard treatment strategies for $\mathrm{NASH}$, while efficient therapeutic agents are not yet available in clinical practice [6].

Gynostemma pentaphyllum (Thunb.) Makino, is a perennial climbing plant of the genus Cucurbitaceae [7]. In China and other Asian countries, Gynostemma pentaphyllum has been widely used for its beneficial pharmacological effects, such as regulating blood lipid and sugar levels as well as anti-inflammatory, hepato-protective, anti-tumor, and immunomodulatory activities [8-11]. The pharmacological impact of Gynostemma pentaphyllum is attributed to the main ingredient, gypenosides (Gyp) [12, 13]. We previously demonstrated that Gyp can be used to treat NASH via the regulation of lipid metabolism [14]. However, their therapeutic impact and mechanism of action require further validation.

Farnesoid X receptor (FXR), a nuclear receptor superfamily member, is important for bile acid and glycolipid metabolism. Kim et al. previously suggested that FXR is a potential target for NAFLD treatment [15]. Moreover, Neuschwander-Tetri et al. demonstrated that a FXR agonist, obeticholic acid (OCA), can significantly improve the pathological outcomes of NASH and thus can be used as a potential treatment [16]. Mounting evidence confirmed that FXR agonists can improve insulin resistance and regulate the glycolipid metabolism [17, 18]. Interestingly, we previously showed that Gyp have beneficial effects in NASH via the improvement of lipid metabolism [14]. Therefore, we used a mouse model of high-fat diet (HFD)-induced NASH to gain mechanistic insights into the impact of Gyp in NASH. Further, we aimed to explore possible crosstalk between Gyp and the FXR-mediated lipid and bile acid metabolic pathways.

\section{Methods}

\section{Laboratory animals and experimental design}

A total of 32 male C57BL/6 specific-pathogen free (SPF) mice, weighing 16-20 g, were purchased from the Nanjing Biomedical Research Institute, Nanjing University (license number: SCXK (Su) 2015-0001). For an acclimation period of 1 week, mice were housed in the animal center of Ningbo University under SPF conditions with food and water available ad libitum. Following the acclimation period, mice were randomly assigned to a control group ( $n=8$ mice) and an experimental group ( $n=24$ mice). Next, mice in the experimental group were fed with a HFD (Research Diets, D12492i, 60\% Kcal fat, energy density: $\mathbf{5 . 2 1} \mathrm{kcal} / \mathrm{g}$, New Brunswick, NJ, USA) for 14 consecutive weeks, while mice in the control group were given a normal control diet (Research Diets, D12450B, 10\% Kcal fat, energy density: $\mathbf{3 . 8 2} \mathrm{kcal} /$ g, New Brunswick, NJ, USA) for 14 consecutive weeks [19]. The ingredients of the normal control diet and HFD are presented in Table 1. At the end of week 10, mice in the experimental group were randomly divided into a HFD group, HFD plus Gyp group, and HFD plus OCA group ( $n=8 /$ group). In the HFD plus Gyp group, mice were given a daily dose of $100 \mathrm{mg} / \mathrm{kg}$ Gyp (Shanghai Ronghe Pharmaceutical Technology Development Co., Ltd., China; batch number: 171019), for 4 consecutive weeks (from week 11 to week 14) as described previously [20]. The fingerprint spectrum of Gyp was analyzed by ultra high-performance liquid chromatography (Thermo Scientific, USA) and quadrupole/electrostatic field orbit trap high-resolution mass spectrometry. Sample preparation and analysis were performed as described previously [21].

In the HFD plus OCA group, mice were given $10 \mathrm{mg} /$ kg/day OCA (BioVision, USA; batch number: 3J28B18980) via oral gavage for 4 consecutive weeks as described previously [22]. Mice in the HFD and control groups received equal volumes of drinking water via oral gavage. The experimental protocol was revised and approved by the Experimental Animal Ethics Committee of Ningbo University. All procedures performed in studies involving animals were in accordance with the ethical standards of the institution or practice at which the studies were conducted.

\section{Specimen collection}

At week 14, mice were fasted for $12 \mathrm{~h}$ and anesthetized with an intraperitoneal injection of $3 \%$ sodium pentobarbital $(3 \mathrm{~mL} / \mathrm{kg})$, and different specimens were collected as described previously [14]. Briefly, blood was collected from the inferior vena cava and centrifuged, and serum samples were collected and stored at $-70{ }^{\circ} \mathrm{C}$ until the detection of biochemical biomarkers. Two liver tissue specimens were extracted from the same liver lobe and placed in either $10 \%$ neutral formalin buffer and paraffin embedded for hematoxylin and eosin (HE) staining or frozen in optimal cutting temperature (OCT) compound for oil red $\mathrm{O}$ staining. Another liver tissue specimen was collected from each mouse and stored at $-70{ }^{\circ} \mathrm{C}$ for subsequent RT-PCR and western blot analyses. The total 
Table 1 Ingredients of the normal and high-fat diets

\begin{tabular}{lll}
\hline Ingredient & Normal control diet $(\mathrm{g} / \mathrm{kg})$ & High-fat diet $(\mathrm{g} / \mathrm{kg})$ \\
\hline Casein, Lactic, 30 Mesh & 200 & 200 \\
Cystine, L & 3 & 3 \\
Lodex 10 & 35 & 125 \\
Sucrose, Fine Granulated & 354 & 72.8 \\
Starch, Corn & 314 & - \\
Solka Floc, FCC200 & 50 & 50 \\
Lard & 20 & 245 \\
Soybean Oil, USP & 25 & 25 \\
S10026B & 50 & 50 \\
Choline Bitartrate & 2 & 2 \\
V1001C & 1 & 1 \\
Dye, Blue FD\&C \#1, Alum. Lake 35-42\% & 0.05 & 0.05 \\
\hline
\end{tabular}

Notes: The energy densities of the normal control diet and high-fat diet were $3.82 \mathrm{kcal} / \mathrm{g}$ and $5.21 \mathrm{kcal} / \mathrm{g}$, respectively

mouse body weight and liver wet weight were recorded, and the liver index was calculated according to the following formula: liver index =liver wet weight/body weight * $100 \%$.

\section{Histopathological examination}

HE staining was performed on 4- $\mu$ m-thick sections according to the standard published protocols [23]. Sections were examined by experienced liver pathologists. The NAFLD activity score (NAS) was determined as described previously according to the following score [24]: steatosis $(0=<5 \% ; 1=5-33 \% ; 2=33-66 \% ; 3=>66 \%)$; intralobular inflammation $(0=$ no lesions; $1=<2$ lesions $/$ field of view; $2=2-4$ lesions/field of view; $3=>4$ lesions/field of view; and ballooning degeneration $(0=$ none; $1=$ rare new balloon cells; $2=$ common new balloon cells).

Frozen liver tissues were sectioned at $10-\mu \mathrm{m}$ thickness on a cryostat (Leica CM1850, Germany), and fat cells were detected by an oil red $\mathrm{O}$ staining kit (Nanjing Jiancheng Bioengineering Institute, Jiangsu, China; batch number: 20180704) following the manufacturer's instructions. Next, sections were analyzed under an inverted fluorescence microscope (Leica 37XB, Germany) and photographed.

\section{Biochemical parameters}

The liver triglyceride (TG) content was analyzed with a TG assay kit (Zhejiang Dongou Diagnostics Co. Ltd. Zhejiang, China, batch number: 201804007) according to the manufacturer's protocols. Specimen preparation and homogenization were carried out as previously detailed [14].

Serum alanine aminotransferase (ALT), aspartate aminotransferase (AST), and fasting blood glucose (FBG) levels were detected using standard kits according to the manufacturer's protocols (Nanjing Jiancheng Bioengineering Institute, Jiangsu, China; batch numbers: 20180629, 20180629 and 20180402137, respectively).

A serum TG kit was obtained from Zhejiang Dongou Diagnostics Co. Ltd. (batch number: 201004007; Zhejiang, China). Kits for the detection of total cholesterol (TC; batch number: 20180526), low-density lipoprotein cholesterol (LDL-C; batch number: 20180705), and highdensity lipoprotein cholesterol (HDL-C; batch number: 20180706) were purchased from Nanjing Jiancheng Bioengineering Institute (Jiangsu, China). All parameters were detected following the manufacturers' protocols on a microplate reader (BioTek, MQX200R).

The fasting insulin (FINS) levels were detected in mice sera samples using a mouse insulin enzyme-linked immunosorbent assay (ELISA) kit (Crystal Chem, Inc., IL, USA; batch number: 18 MAUMI477A and cat. no. 90080) following the manufacturer's instructions. The absorbance was measured within $30 \mathrm{~min}$, and the insulin concentration in each sample was calculated using a standard curve. For each mouse, the homeostasis model assessment-insulin resistance (HOMA-IR) index was estimated using: HOMA-IR = FBG $\times$ FINS/22.5 [25].

\section{Quantitative bile acid profiling}

A total of $10 \mathrm{mg}$ liver tissue was cut and transferred to a microcentrifuge tube. Also, $25 \mathrm{mg}$ beads and $20 \mu \mathrm{l}$ ultrapure water were mixed using a homogenizer (BB24, Next Advance, Inc., Averill Park, NY, USA). Samples were mixed with $180 \mu \mathrm{l}$ acetonitrile/methanol (8:2) containing 10 internal standards and centrifuged at 13000 $\mathrm{rpm}$ for $20 \mathrm{~min}$ and $4{ }^{\circ} \mathrm{C}$ (Microfuge 20R, Beckman Coulter, Inc., Indianapolis, IN, USA). Following centrifugation, the supernatant was transferred to a 96-well plate and freeze-dried with FreeZone freeze dryer (Labconco, Kansas City, MO, USA). The dried sample powder and 
freeze-dried standard were remixed with 1:1 (v/v) acetonitrile/methanol $(80 / 20, \mathrm{v} / \mathrm{v})$ and ultrapure water, and then centrifuged at $13000 \mathrm{rpm}$ for $20 \mathrm{~min}$ and $4{ }^{\circ} \mathrm{C}$ (Microfuge 20R). A total of $5 \mu \mathrm{l}$ supernatant was transferred to a 96well plate and then subjected to LC-MS analysis.

Bile acids were analyzed using an ultra-performance liquid chromatography tandem mass spectrometry (UPLC-MS/MS) system (ACQUITY UPLC-Xevo TQ-S, Waters Corp., Milford, MA, USA), in accordance with previous reports $[26,27]$.

\section{Real-time quantitative polymerase chain reaction}

Total RNA was extracted from the frozen liver tissues using an RNA extraction kit (Sangon Biotech Co., Ltd., China; batch number: E928KA9723) following the manufacturer's instructions. The RNA concentration was estimated at $260 \mathrm{~nm}$ on an Infinite 200 PRO microplate reader (Tecan Group Ltd., Switzerland). Different PCR primers were designed by BioTNT (Shanghai, China) (Table 2). Reverse transcription was achieved using an iScript ${ }^{\mathrm{Tm}}$ cDNA Synthesis Kit (BioRad, Hercules, CA, USA; cat. no. 170-8891). Realtime PCR was performed using a TB Green ${ }^{\text {Th }}$ Premix Ex $\operatorname{Taq}^{\mathrm{Tm}}$ (TaKaRa, Janpan, cat. no. RR420A) and a QuantStudio $^{\text {Tx }}$ real-time PCR system (Applied Biosystem, Foster City, CA, USA). $\beta$-Actin served as the internal control.

\section{Western blotting}

Total proteins were extracted from $50 \mathrm{mg}$ of liver tissue with $600 \mu \mathrm{l}$ radioimmunoprecipitation assay (RIPA) lysis buffer (Beyotime,China; cat. no. P0013B) supplemented with protease and phosphatase inhibitors (Beyotime; cat. no. P1045-1 and P1045-2, respectively). The mixture was homogenized twice at $65 \mathrm{~Hz}$ for $1 \mathrm{~min}$ on an automatic sample rapid grinder (Shanghai Jingxin Industrial Development Co., Ltd. Company, model: JXFSTPR-24). Next, the mixture was centrifuged at $12000 \mathrm{rpm}$ for $15 \mathrm{~min}$ at $4{ }^{\circ} \mathrm{C}$, and the middle layer was extracted with a $1-\mathrm{ml}$ syringe. The upper fat layer and the pellet were discarded. The protein concentration was estimated using a Pierce ${ }^{\mathrm{Tm}}$ BCA Protein Assay Kit (Thermo Fisher, cat. no. TI269557).

Proteins were separated using $10 \%$ or $12 \%$ sodium dodecyl sulfate polyacrylamide gel electrophoresis (SDSPAGE) gels and sequentially transferred onto Immobilon-FL Transfer membranes. Following blocking with blocking buffer (Odyssey, cat no. 927-40,000), membranes were incubated with the primary antibodies at $4{ }^{\circ} \mathrm{C}$ overnight. The following primary antibodies were used in this study: anti-FXR monoclonal antibody (Thermo Fisher, A9033A; 1:1000); anti-NROB2 antibody (Abcam, Cambridge, MA, USA; ab186874;1:500); antiCYP7A1 antibody (Abcam, ab65596, 1:1000); anti-bile salt export pump (BSEP) polyclonal antibody (Thermo Fisher, PA5-78690, 1:2000); anti- $\mathrm{Na}^{+}$-taurocholate cotransporting polypeptide (NTCP) polyclonal antibody

Table 2 Primer sequences used for real-time polymerase chain reaction

\begin{tabular}{|c|c|c|c|}
\hline Gene & Gene ID & Forward primer ( $5^{\prime}$ to $\left.3^{\prime}\right)$ & Reverse primer (5' to $3^{\prime}$ ) \\
\hline FXR & 20186 & CAGATTTCCTCCTCGTCTTAC & CCTGAGTTCATAGATGCCA \\
\hline SHP & 23957 & TTCCTTGCTTTGGATACAGTG & GAGGTTTGGGGAGTCATCA \\
\hline SREBP1 & 20787 & GGCTTGGTGATGCTATGTTGA & GGCAAAGGAACAACTGAGACC \\
\hline FASN & 14104 & GTGTGGTGGGTT TGG TGA AT & AGATGTGTTGCT GAG GTT GGA \\
\hline SCD1 & 20249 & TGAGGG GCA GTG TCT GTA A & GGAAACTAAAGCAATGGA TGG \\
\hline PPARa & 19013 & GAGGATGGGGACTITGTTCT & GGC TIT TTG GCT GTA GGA GG \\
\hline CPT1 & 12894 & ACC CAG TCA GAT TCC AAC C & ACAAAGCACCCA TTA CTT GAG \\
\hline MTTP & 17777 & CCACCAGAATCGTAAGGTTCA & GGACAGCAGGAT GTT CTT CAC \\
\hline CYP7A1 & 13122 & GTACCTTGATGAAAGTGGGAA & ATTGCT TGA TTT CTT GGA CAG \\
\hline FGFR4 & 14186 & СCT TCT GTT CCA GCC TTA TG & TTC TCT GAG GAT GAG TCC AAG \\
\hline BSEP & 27413 & AAT GTT CAG TTC CTC CGT TC & GTC CCC ATA CTT GAT GTT GTC \\
\hline NTCP & 20493 & GTCATCAATGTGGGCAACAGC A & TGAAAGGCATCAGGGAGGAGG TA \\
\hline KLB & 83379 & СCT TCT GTT CCA GCC TTA TG & TTC TCT GAG GAT GAG TCC AAG \\
\hline FGF15 & 14170 & GAAGCCAGA AGG TAT GAA GTC & CCA AGT TTG TAA CCC CAG T \\
\hline$\beta$-Actin & 81822 & CCT CTA TGC CAA CAC AGT & AGC CAC CAA TCC ACA CAG \\
\hline LPL & 16956 & CGA GAGCGAGAA CAT TCC CT & TGT CCA CCT CCG TGT AAA TCA A \\
\hline
\end{tabular}


(Thermo Fisher, PA5-80001, 1:2000); anti-fibroblast growth factor 15 (FGF15) antibody (Abcam; ab229630, 1:1000); anti-FGF receptor 4 (FGFR4) antibody (Abcam, ab119378,1:500); anti-Klotho beta (KLB) antibody (Abcam, ab233416, 1:1000); anti-sterol regulatory element-binding protein 1 (SREBP1) antibody (Abcam, ab28481, 1:1000); anti-SCD1 antibody (Abcam,ab19862, 1:1000); anti-fatty acid synthetase antibody (Abcam, ab22759, 1:1000); anti-peroxisome proliferator-activated receptor alpha $(\mathrm{PPAR} \alpha)$ antibody (Abcam, ab61182, 1: 500); anti-carnitine palmitoyl transferase 1A (CPT1A) antibody (Abcam, ab128568, 1:1000); anti-lipoprotein lipase (LPL) antibody (Abcam, ab21356, 1:1000); antimicrosomal triglyceride transfer protein (MTTP) antibody (Abcam, ab75136, 1:500), and anti-glyceraldehyde3-phosphate dehydrogenase (GAPDH) rabbit polyclonal antibody (Proteintech Group, Inc., 10,494-1-AP, 1: 1000). The next day, membranes incubated with horseradish peroxidase (HRP)-conjugated goat anti-mouse IgG (Beijing Dingguo Changsheng Biotechnology Co., IH-0031) or HRP-conjugated goat anti-rabbit IgG (Beijing Dingguo Changsheng Biotechnology Co., IH0011) for $1 \mathrm{~h}$ at room temperature. BeyoECL Plus A (Beyotime, P0018 M-1) and BeyoECL Plus B (Beyotime, P0018 M-2) were used for development, and chemiluminescence was used for visualization. Chemographic analysis software (ChemiScope 3000 mini, Shanghai Qinxiang Scientific Instrument Co., Ltd) was used to analyze the grey values of each band.

\section{Statistical analysis}

The data are presented as the mean \pm standard deviation. One-way analysis of variance (least significance difference $[L S D]$ test) was used to compare the data for different groups. All statistical analyses were performed using SPSS software version 16.0 (SPSS, Inc., Chicago, IL USA). A $P$ value $<0.05$ was considered to be statistically significant.

\section{Results}

\section{Dietary intake and activity level of mice}

Our results demonstrated that the dietary intake of mice in the HFD group was higher than that of mice in control group $(P<0.01)$. There were no significant differences in dietary intake among the HFD group, Gyp- treated group and OCA-treated group $(P>0.05$; Table 3).

Further, during the experimental phase, we observed a phenomenon that mice in the HFD group were relatively lazy and showed reduced activity compared to mice in the control group. On the other hand, the mice in the Gyp-treated group and the OCA-treated group were relatively active compared to mice in the HFD group.

\section{Analysis of gyp}

High-performance liquid chromatography and mass spectrometric analysis of the Gyp fingerprint spectrum revealed that the contents of gypenoside XLIX (molecular formula: C52H86O21, molecular weight: 1047.23) and gypenoside A (molecular formula: C46H74O17, molecular weight: 898.49 ) were $574.91 \mathrm{mg} / \mathrm{g}$ and $342.66 \mathrm{mg} /$ g, respectively (Fig. 1).

\section{Impact of gyp treatment on the liver index and mice weight}

Compared to the control group, mice in the HFD group had a significantly higher body weight starting from weeks 10-14 (Fig. 2a, $P<0.01$ ). In the Gyp- and OCAtreated groups, the mice weight was significantly lower than that in the HFD group at week 12 (Fig. 2a, $P<$ 0.01 ). Interestingly, there was no significant difference in body weight between the Gyp group and the OCAtreated group (Fig. 2a, $P>0.05$ ).

The liver wet weight and liver index in the HFD group were significantly higher than those in the control group $(P<0.01)$. In the Gyp-treated and the OCA-treated groups, the wet weight of livers as well as the liver indices were significantly lower than those of the HFD group (Fig. $2 \mathrm{~b}$ and $\mathrm{c}, P<0.01$ ). There were no significant differences in the liver wet weight and liver index between the Gyp-treated group and the OCA-treated group (Fig. 2b and c, $P>0.05)$.

\section{Gyp treatment improved hepatic steatosis}

In control mice, HE staining revealed typical liver histology without signs of inflammation or accumulation of lipid droplets in the hepatocytes (Fig. 3A1). Following HFD feeding, mice in the HFD group demonstrated signs of obvious hepatic steatosis accompanied by scattered inflammation and ballooning degeneration (Fig.

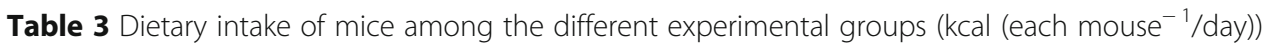

\begin{tabular}{|c|c|c|c|c|c|}
\hline Group & $n$ & 11 week & 12 week & 13 week & 14 week \\
\hline C & 8 & $8.94 \pm 0.16$ & $8.85 \pm 0.21$ & $9.21 \pm 0.25$ & $8.95 \pm 0.13$ \\
\hline HFD & 8 & $12.20 \pm 0.22^{* *}$ & $12.10 \pm 0.39^{* *}$ & $12.41 \pm 0.25^{* *}$ & $12.24 \pm 0.28^{* *}$ \\
\hline HFD + Gyp & 8 & $12.10 \pm 0.34$ & $12.00 \pm 0.38$ & $12.32 \pm 0.13$ & $12.25 \pm 0.20$ \\
\hline $\mathrm{HFD}+\mathrm{OCA}$ & 8 & $12.02 \pm 0.23$ & $12.09 \pm 0.38$ & $12.42 \pm 0.36$ & $12.18 \pm 0.21$ \\
\hline
\end{tabular}

${ }^{* *} P<0.01$ vs $C$ group. $C$ control, HFD high-fat diet, Gyp gypenosides, OCA obeticholic acid 


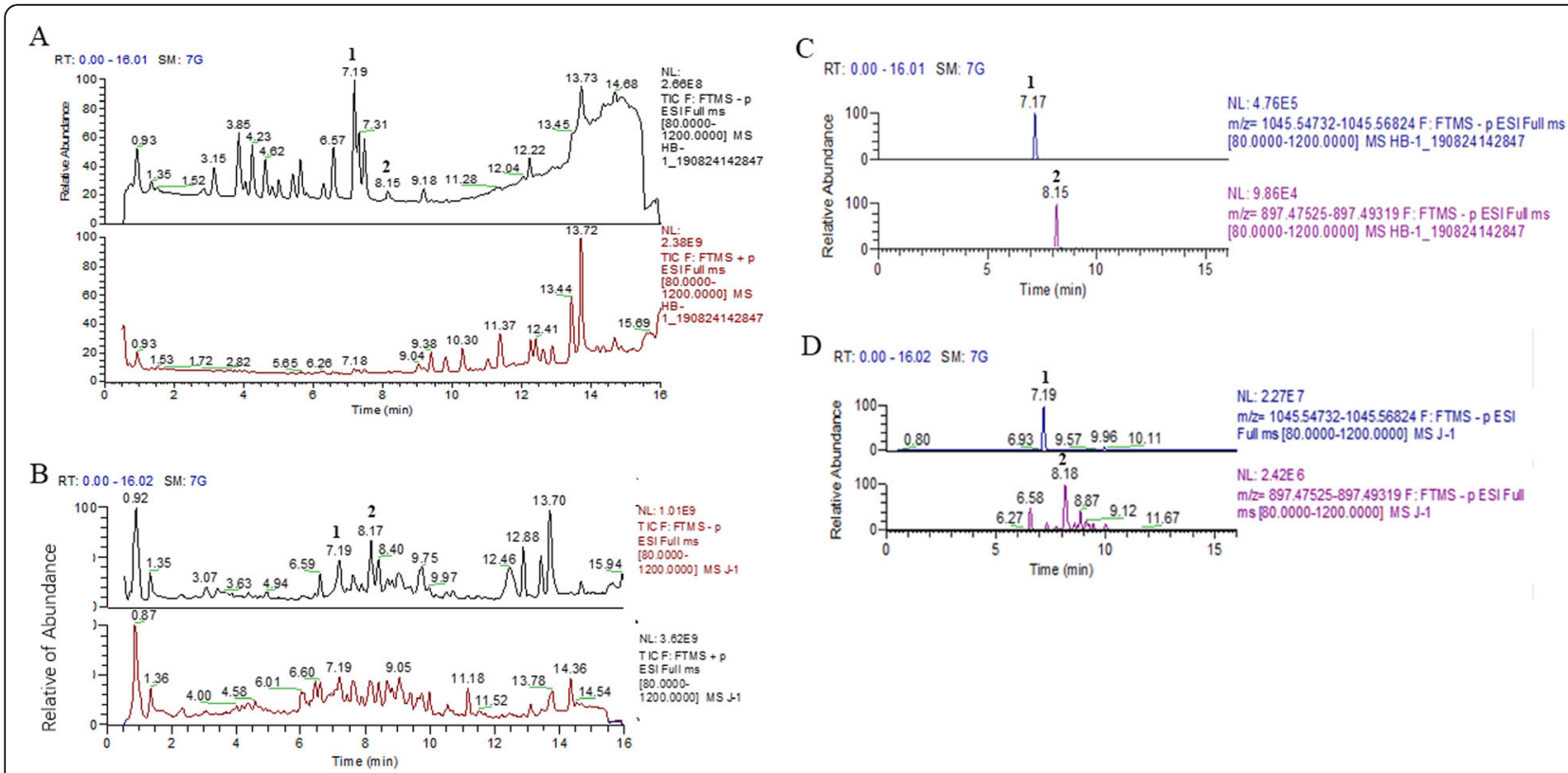

Fig. 1 Total-ion chromatograms and select-ion chromatograms of reference standard and gypenosides. a Total-ion chromatograms of reference standard; b Total-ion chromatograms of gypenosides sample; c Select-ion chromatograms of reference standard; $\mathbf{d}$ Select-ion chromatograms of gypenosides sample; 1, gypenosides XLIX; 2, Gypenosides A

3A2). Particularly, hepatocytes appeared swollen with a large number of lipid droplets accumulated in the cytoplasm. Similarly, oil red O staining verified the accumulation of large lipid droplets in the hepatocytes with dark red intracellular staining in the HFD group mice (Fig. 3B2). These results confirmed that HFD feeding for 14 weeks induced NASH. These pathological changes were significantly alleviated after treatment with Gyp or OCA (Fig. 3A3 and A4, respectively). Further, oil red O staining confirmed the alleviation of hepatic steatosis signs in the Gyp-treated group and the OCA-treated group (Fig. $3 \mathrm{~B} 3$ and $\mathrm{B} 4$, respectively).

Next, we examined the liver TG levels and NAS scores among mice from the different experimental groups. Compared to the control group, mice in the HFD group had significantly higher liver TG levels and NAS scores (Fig. $4 \mathrm{a}$ and $\mathrm{b}, P<0.01$ ). In the Gyp-treated and OCA- treated groups, the liver TG and NAS scores were significantly lower than those in the HFD group (Fig. 4a and $\mathrm{b}, P<0.01)$. Interestingly, the liver TG levels were significantly lower in the Gyp-treated group than in the OCA-treated group (Fig. $4 \mathrm{a}, P<0.05$ ), while the difference in NAS scores was not significant (Fig. $4 \mathrm{~b}, P>$ 0.05).

\section{Gyp treatment improved serum biochemical parameters}

We examined the impact of HFD feeding and Gyp treatment on different biochemical parameters in serum samples. In the HFD group, serum ALT and AST levels were significantly higher than those of the control group (Fig. 5a and b, $P<0.01$ ). Treatment with Gyp significantly decreased the serum ALT and AST activities, while treatment with OCA significantly decreased the serum ALT activities (Fig. 5a and b, $P<0.05$ ). Moreover,
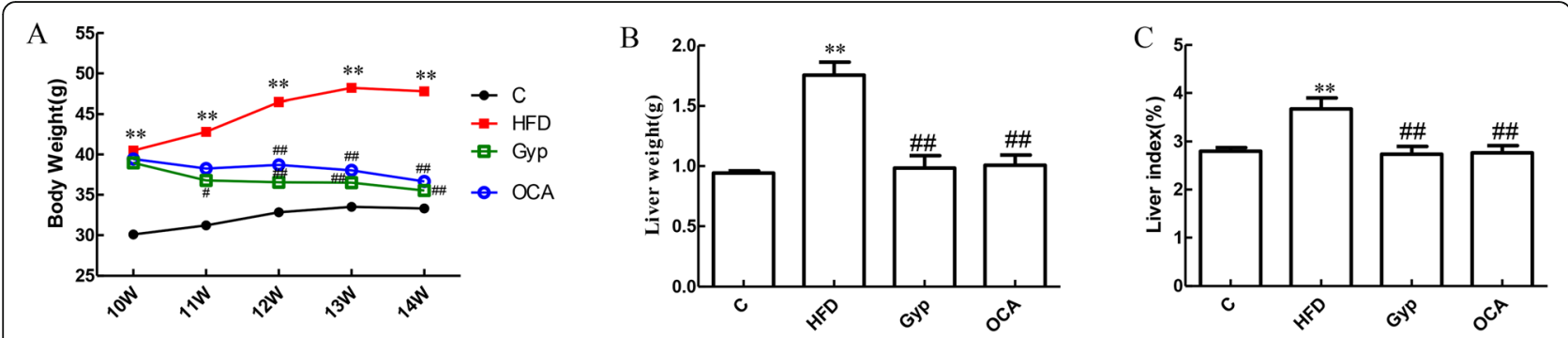

Fig. 2 Alterations in body weight, liver wet weight, and liver index in the different experimental groups. a Body weight, $\mathbf{b}$ liver wet weight, and $\mathbf{c}$ liver index. ${ }^{* *} P<0.01$, vs control group; ${ }^{\# \# P}<0.01,{ }^{\#} P<0.05$, vs HFD group. C, control; HFD, high-fat diet; Gyp, gypenosides; OCA, obeticholic acid 


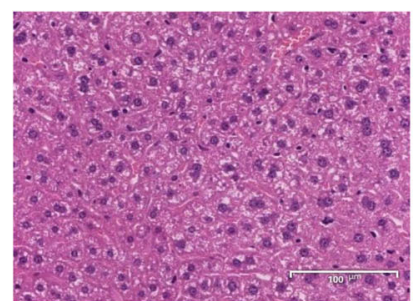

(A1)

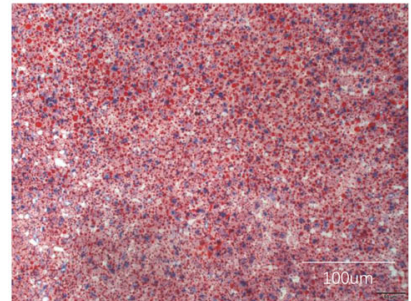

(B1)

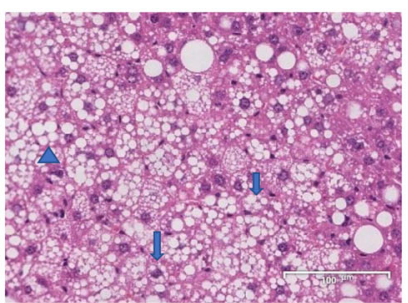

(A2)

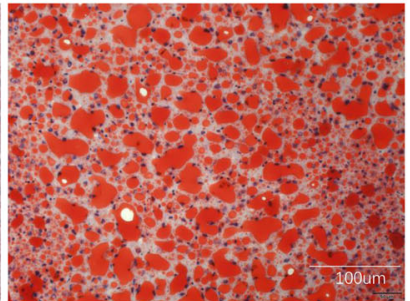

(B2)

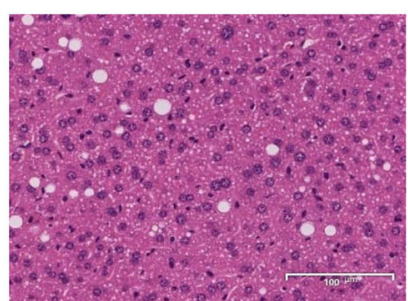

(A3)

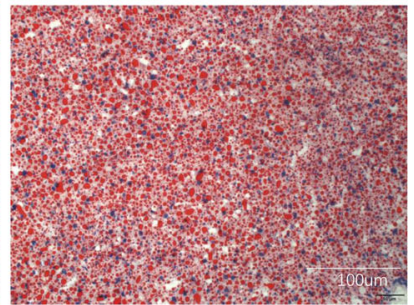

(B3)

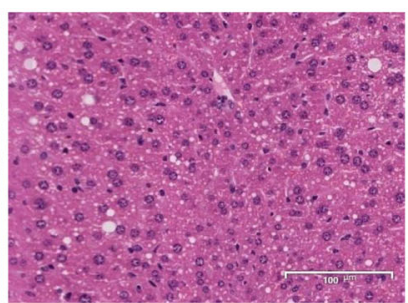

(A4)

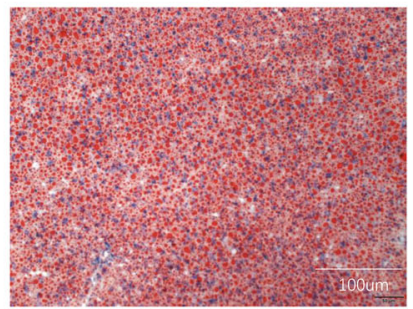

(B4)

Fig. 3 Representative images of pathological changes in liver tissues among the different experimental groups. A: Hematoxylin and eosin staining, B: Oil red O staining. 1: Control group, 2: high-fat diet group, 3: gypenosides group, 4: obeticholic acid group, scale bar $=100 \mu m, n=8$. The triangular shape indicates steatosis, and an arrow indicates a balloon-like change

compared with the control diet, HFD feeding for 14 weeks caused a significant increase in the serum FINS and FBG levels as well as the HOMA-IR index (Fig. 5ce, $P<0.01)$. In the treated groups, the serum FINS and FBG levels as well as the HOMA-IR were significantly lower in the Gyp-treated and OCA-treated groups compared with the HFD group (Fig. 5c-e, $P<0.01$ ). Notably, no significant differences were observed in the serum FINS and FBG levels or the HOMA-IR between the Gyp-treated and OCA-treated groups (Fig. 5c-e, $P>0.05)$.

In the HFD group, serum TG, TC, and LDL-C levels were significantly higher than those of the control group (Fig. 5f-h, $P<0.01$ ). Additionally, the serum TC and LDL-C levels were significantly lower in the Gyp- and OCA-treated groups compared to the HFD group (Fig. $5 \mathrm{~g}$ and $\mathrm{h}, P<0.05)$. There were no significant differences in the TG levels between the Gyp and OCA groups (Fig. 5f) or in the HDL-C levels among the examined groups (Fig. 5i).

\section{Gyp treatment influenced the bile acid profile}

Next, we analyzed the differences in bile acid content among mice in the different experimental groups. Compared with those in the control group, the levels of the total bile acid (TBA), $\alpha$-muricholic acid ( $\alpha \mathrm{MCA}$ ), tauro

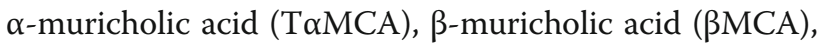
tauro $\beta$-muricholic acid (T $\beta$ MCA), taurochenodeoxycholic acid (TCDCA), cholic acid (CA), glycochenodeoxycholic acid (GCDCA), glycocholic acid (GCA), and taurocholic acid (TCA) were significantly increased in liver tissues from the HFD group (Fig. 6a-e, g, h and $\mathrm{j}-1$, respectively; $P<0.05)$, while alloLCA and $\beta$ CDCA levels were significantly decreased (Fig. $6 \mathrm{f}$ and $\mathrm{i}$, respectively; $P<0.05)$. Compared with those in the HFD group, the TBA, TaMCA, TßMCA, TCDCA, CA, GCDCA, GCA,
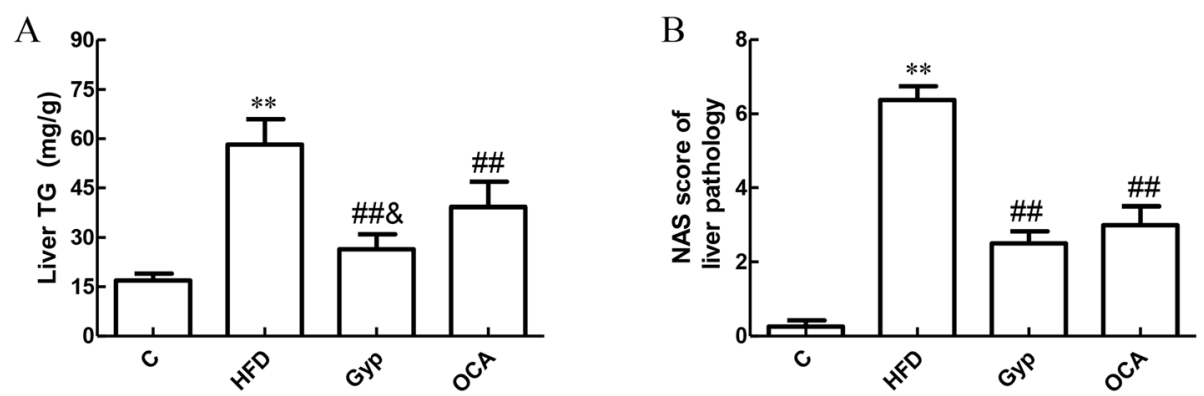

Fig. 4 Changes in liver TG and the NAS among the different experimental groups: a Liver TG, b NAS score. ${ }^{*} P<0.01$, vs control group; ${ }^{\# \#} P<0.01$, ${ }^{\#} P<0.05$, vs HFD group; ${ }^{2} P<0.05$, vs the OCA group, $n=8$. TG, triglycerides; NAS, NAFLD activity score; $C$, control; HFD, high fat diet; Gyp, gypenosides; OCA, obeticholic acid 


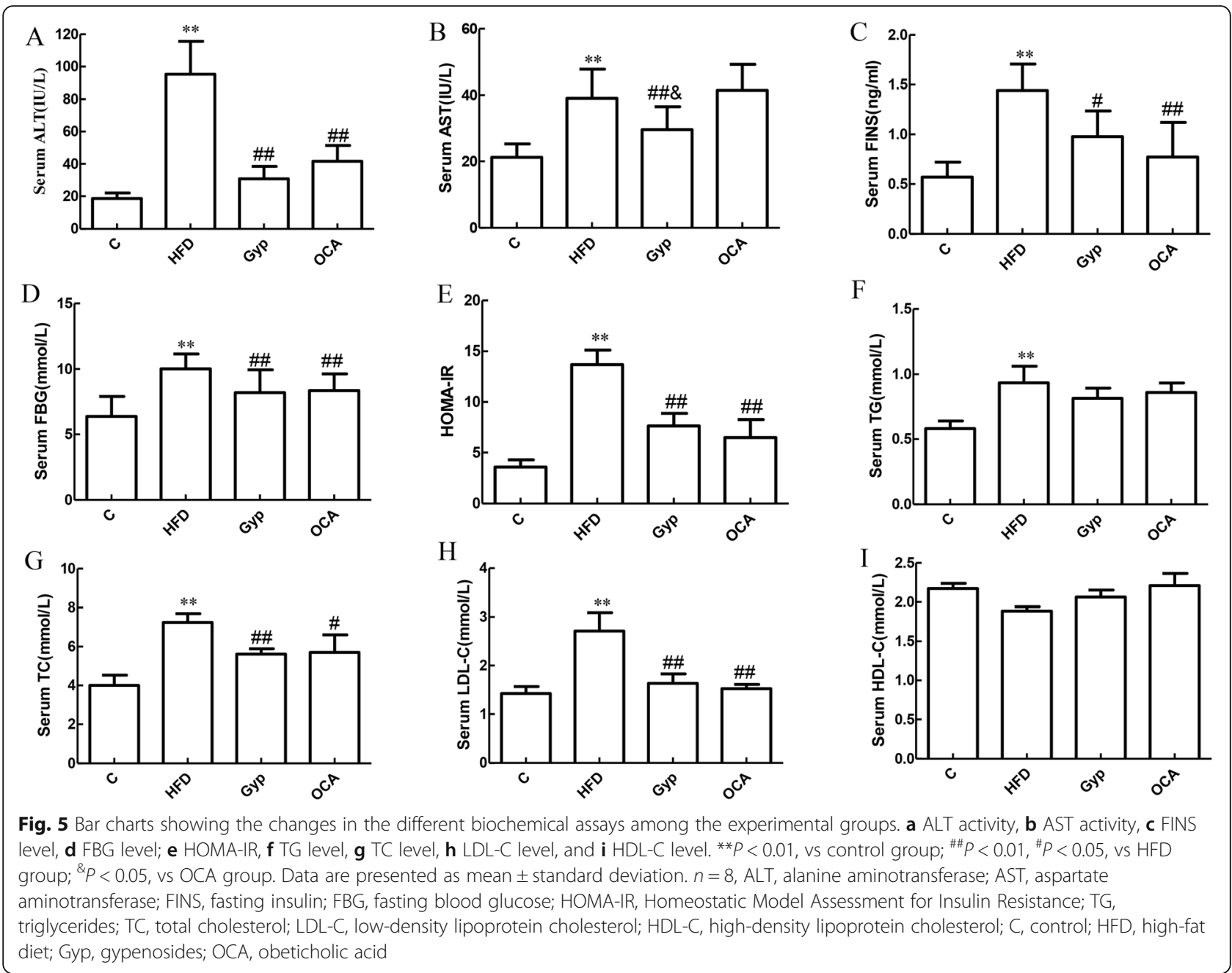

and TCA levels were significantly lower in the Gyptreated and OCA-treated groups (Fig. 6a, d, e, g, h and j$1, P<0.05)$. In addition, the liver contents of aMCA and $\beta M C A$ were significantly lower in the Gyp-treated group than in the HFD group (Fig. $6 \mathrm{~b}$ and $\mathrm{c}, P<0.05$ ). On the other hand, the alloLCA and $\beta C D C A$ levels in the Gyptreated and OCA-treated groups were significantly higher than those in the HFD group (Fig. $6 \mathrm{f}$ and i, respectively, $P<0.05)$.

\section{Gyp increased the FXR and SHP mRNA expression}

To gain mechanistic insights into the signaling pathways responsible for the effects of Gyp in NAFLD, we evaluated the mRNA and protein expression of several molecules associated with lipid metabolism in different liver tissues. In the HFD group, the relative mRNA expression of FXR and SHP as well as the FXR protein level were significantly lower than those of the control group (Fig. $7 \mathrm{a}-\mathrm{d}, P<0.01$ ). The relative FXR and SHP mRNA expression levels as well as the FXR protein level were significantly higher in the Gyp-treated and OCA-treated groups (Fig. $7, P<0.01$ ).

Gyp altered the mRNA expression of SREBP1, SCD1, FASN, PPARa, CPT1, LPL, and MTTP

Compared with those in the control group, the SREBP1, SCD1 and FASN mRNA levels were significantly upregulated (Fig. 8a-c, $P<0.01$ ) and the PPAR $\alpha, C P T 1$, LPL, and MTTP mRNA levels were significantly downregulated in the HFD group (Fig. $8 \mathrm{~d}-\mathrm{g}, P<0.01$ ). In the Gyptreated and OCA-treated groups, the relative mRNA expression levels of SREBP1, SCD1 and FASN were significantly lower than those in the HFD group (Fig. 8a-c, $P<$ 0.01). In contrast, the PPAR $\alpha$, CPT1, LPL, and MTTP mRNA levels in the Gyp-treated group (Fig. 8d-g) and the PPAR $\alpha$, LPL and MTTP mRNA levels in the OCAtreated group (Fig. $8 \mathrm{~d}, \mathrm{f}$ and $\mathrm{g}$ ) were significantly higher than those of the HFD group $(P<0.05)$. Compared to those in the OCA-treated group, the relative mRNA expression levels of PPAR $\alpha$ and CPT1 were significantly higher in the Gyp-treated group (Fig. 8d and e, $P<0.05$ ). 

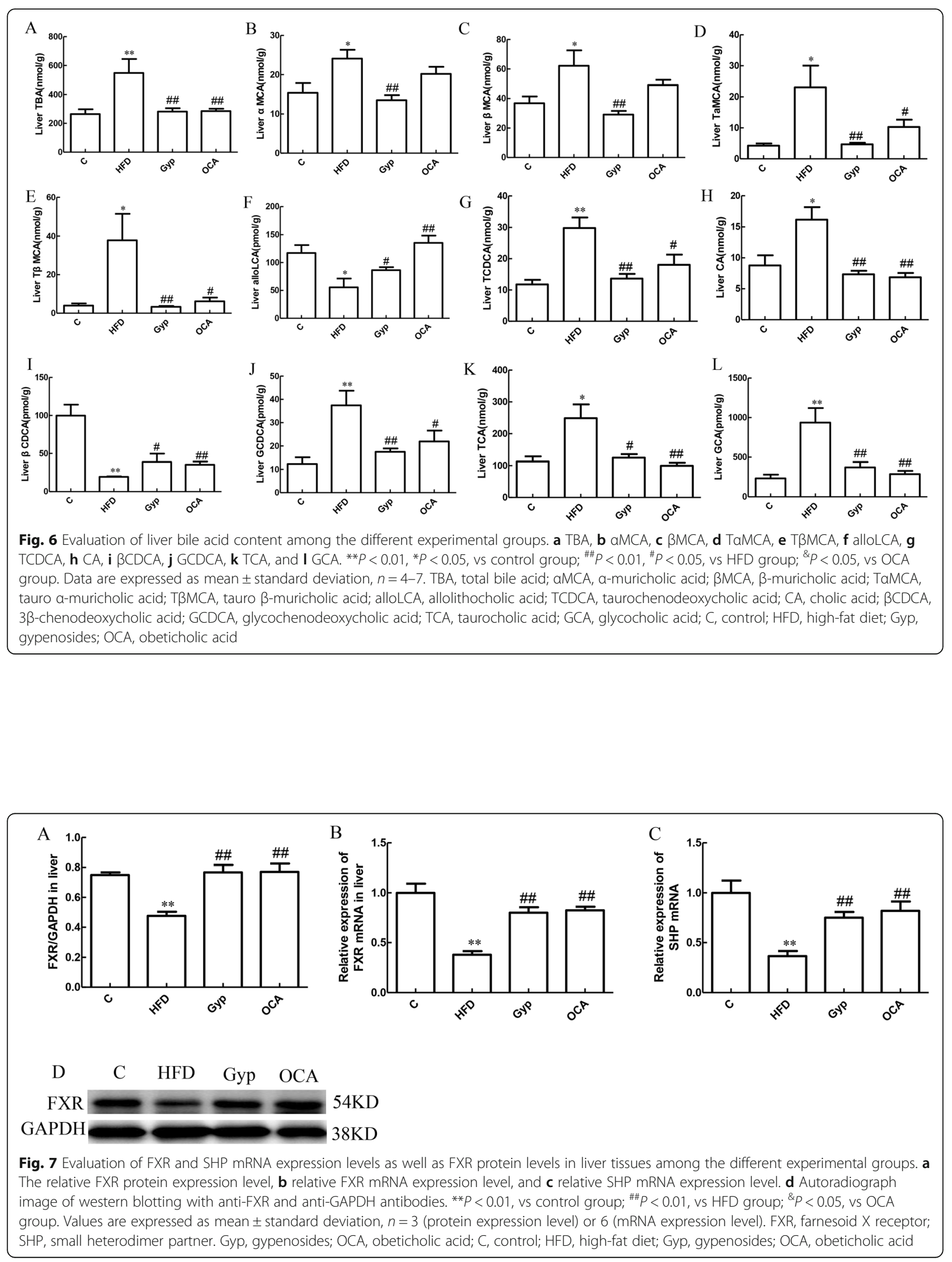


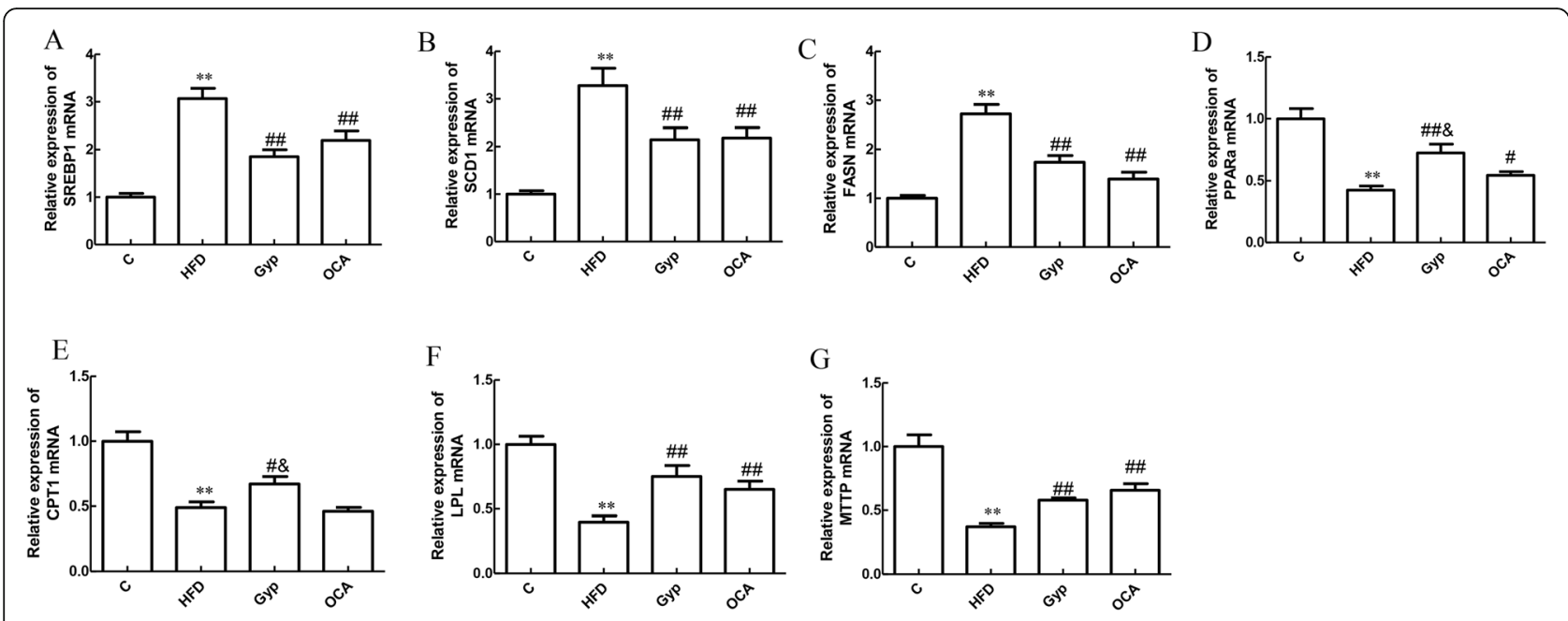

Fig. 8 Analysis of the SREBP1, SCD1, FASN, PPARa, CPT1, LPL, and MTTP mRNA levels in liver tissues among the different experimental groups. a The relative SREBP1 mRNA expression level, $\mathbf{b}$ relative SCD1 mRNA expression level, $\mathbf{c}$ relative FASN mRNA expression level, $\mathbf{d}$ relative PPARa mRNA expression level, e relative CPT1 mRNA expression level, $\mathbf{f}$ relative LPL mRNA expression level, and $\mathbf{g}$ relative MTTP mRNA expression level. ${ }^{*} P<0.01$, vs control group; ${ }^{\# \#} P<0.01,{ }^{\#} P<0.05$, vs HFD group; ${ }^{\&} P<0.05$, vs OCA group. Data are presented as mean \pm standard deviation and are the results of 2 independent experiments, $n=6$. SREBP1, sterol-regulatory element-binding protein 1; SCD1, stearyl coenzyme A desaturation enzyme 1; FASN, fatty acid synthetase; PPARa, peroxisome proliferator-activated receptor alpha; CPT1, carnitine palmitoyl transferase 1; LPL, lipoprotein lipase; MTTP, microsomal triglyceride transfer protein; C, control; HFD, high fat diet; Gyp, gypenosides; OCA, obeticholic acid

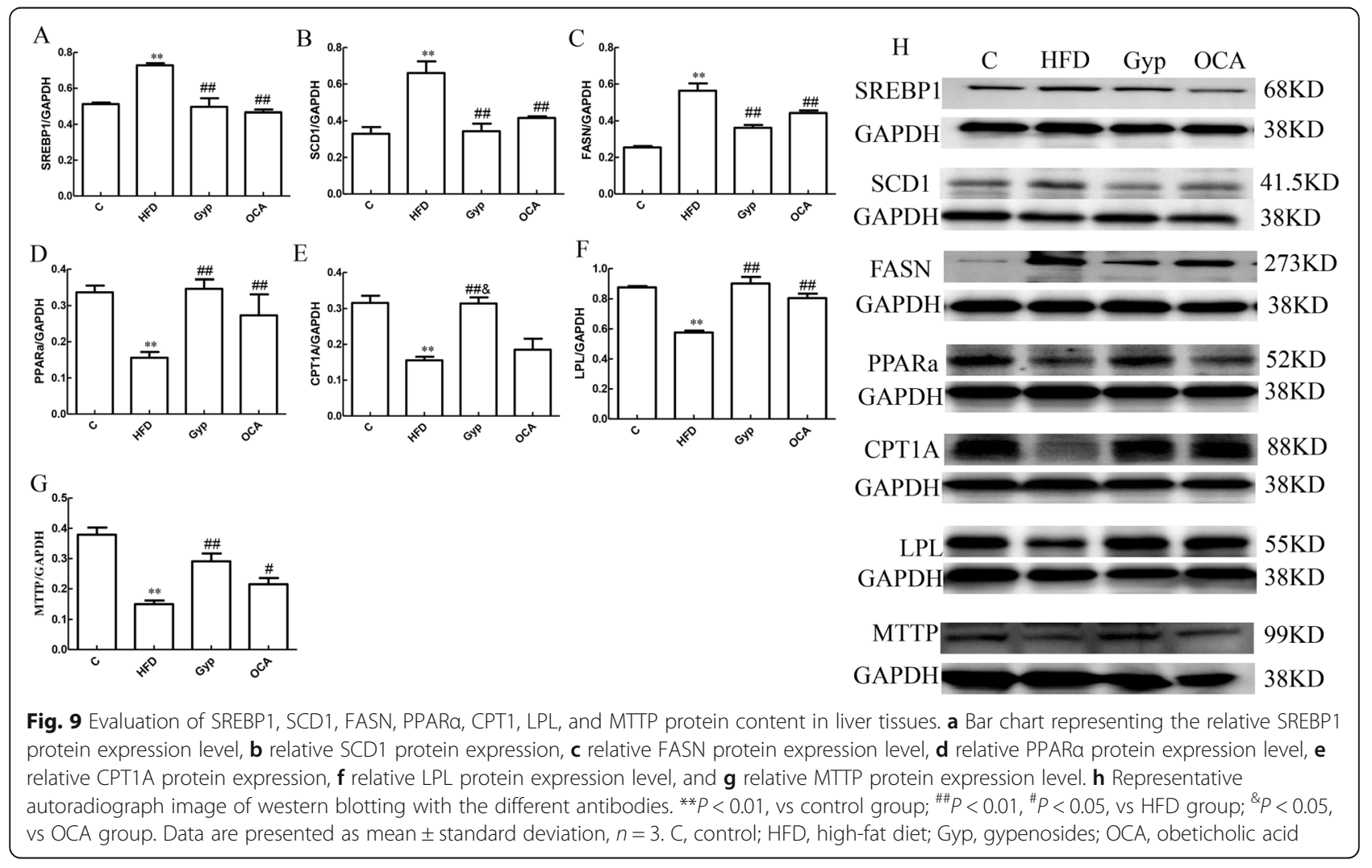


Gyp altered the protein levels of SREBP1, SCD1, FASN, PPARa, CPT1, LPL, and MTTP

The relative expression levels of SREBP1, SCD1 and FASN proteins were significantly higher in the HFD group than the control group (Fig. $9 \mathrm{a}-\mathrm{c}$ and $\mathrm{h}, P<0.01$ ). While the relative protein expression levels of PPAR $\alpha$, CPT1, LPL and MTTP were decreased significantly in the HFD group (Fig. 9d-g and h, $P<0.01$ ). In the Gyptreated and OCA-treated groups, the relative expression levels of |SREBP1, SCD1, and FASN proteins were significantly lower than those in the HFD group (Fig. 9a-c and $\mathrm{h}, P<0.01)$. The PPAR $\alpha, \mathrm{CPT} 1, \mathrm{LPL}$ and MTTP protein levels in the Gyp-treated group and the PPAR $\alpha$, LPL and MTTP protein levels in the OCA-treated group were significantly upregulated compared to the HFD group (Fig. 9, $P<0.05$ ).

\section{Gyp altered FGF15, CYP7A1, and BSEP expression}

Compared to the control group, the relative expression of CYP7A1 mRNA was significantly increased in the HFD group (Fig. 10a, $P<0.01$ ). In contrast, the relative mRNA expression of FGF15 and BSEP was significantly lower in the HFD group (Fig. 10c and d, $P<0.01$ ). There were no significant differences in the relative FGFR4, KLB, and NTCP mRNA levels between the control group and the HFD group (Fig. 10b, e and f, $P>0.05$ ). In the Gyp-treated and OCA-treated groups, the relative
FGF15 and BSEP mRNA expression levels were significantly higher than those in the HFD group (Fig. 10c and d, $P<0.05)$, whereas the CYP7A1 mRNA level was significantly lower (Fig. 10a, $P<0.01$ ).

\section{Gyp influenced the protein expression of FGF15, CYP7A1, and BSEP}

To further investigate the underlying signaling pathway, we analyzed the protein expression levels of FGF15, FGFR4, KLB, CYP7A1, NTCP, and BSEP in liver tissues collected from the different experimental groups (Fig. 11). CYP7A1 protein expression was significantly higher in the HFD group than in the control group (Fig. 11a, $P<0.01$ ). Compared with those in the control group, the relative expression levels of FGF15 and BSEP proteins were significantly decreased in the HFD group (Fig. 11c and d, $P<0.01$ ). The relative expression levels of CYP7A1 protein were significantly lower in the Gyp-treated and OCA-treated groups than in the HFD group (Fig. 11a, $P<0.01$ ). In contrast, the relative expression levels of FGF15 and BSEP proteins were significantly higher in the Gyptreated and OCA-treated groups (Fig. 11c and d, $P<$ 0.05). The FGFR4, KLB, and NTCP protein levels did not differ significantly among the different experimental groups (Fig. 11b, e and f, $P>0.05$ ).

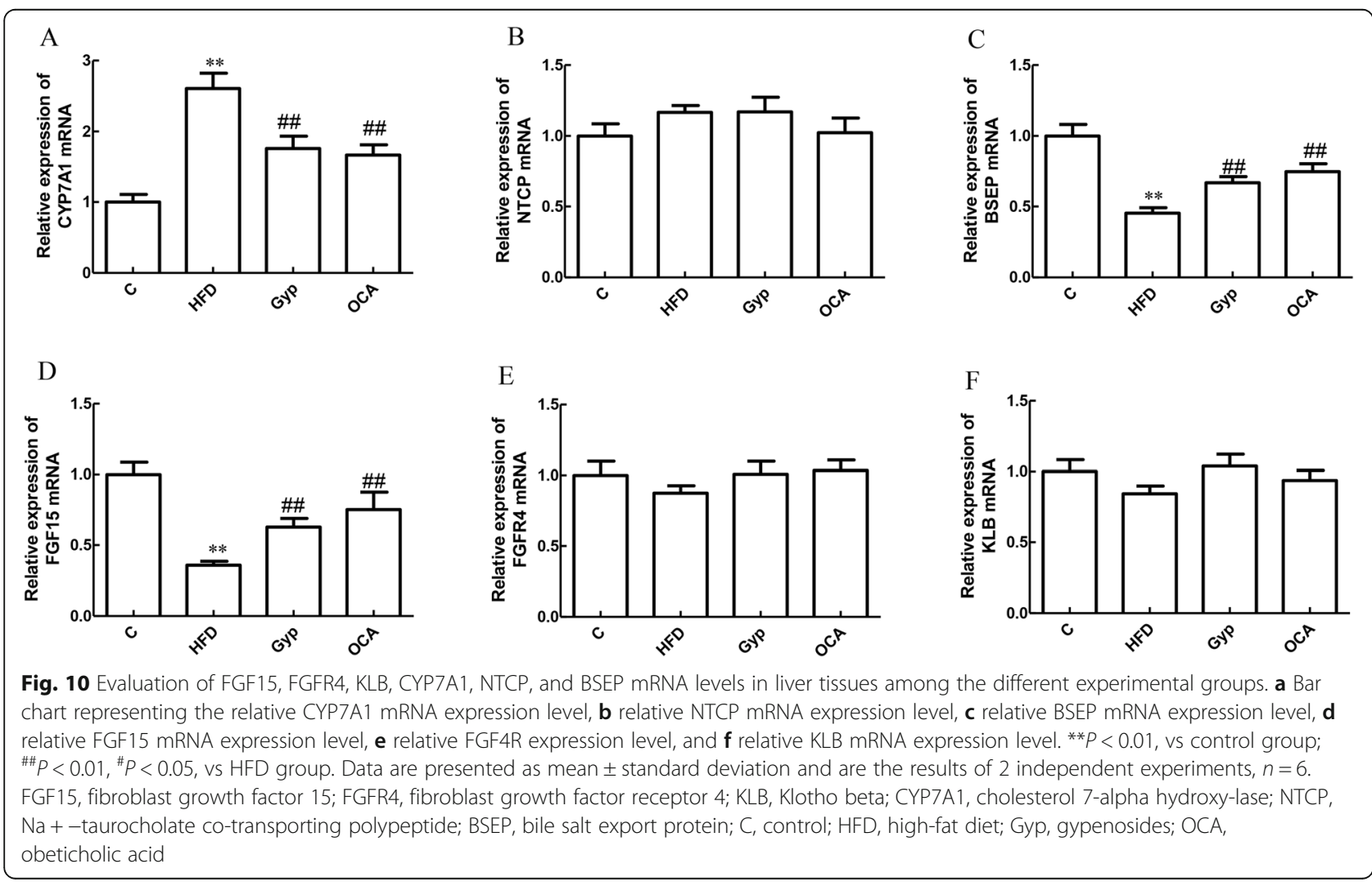




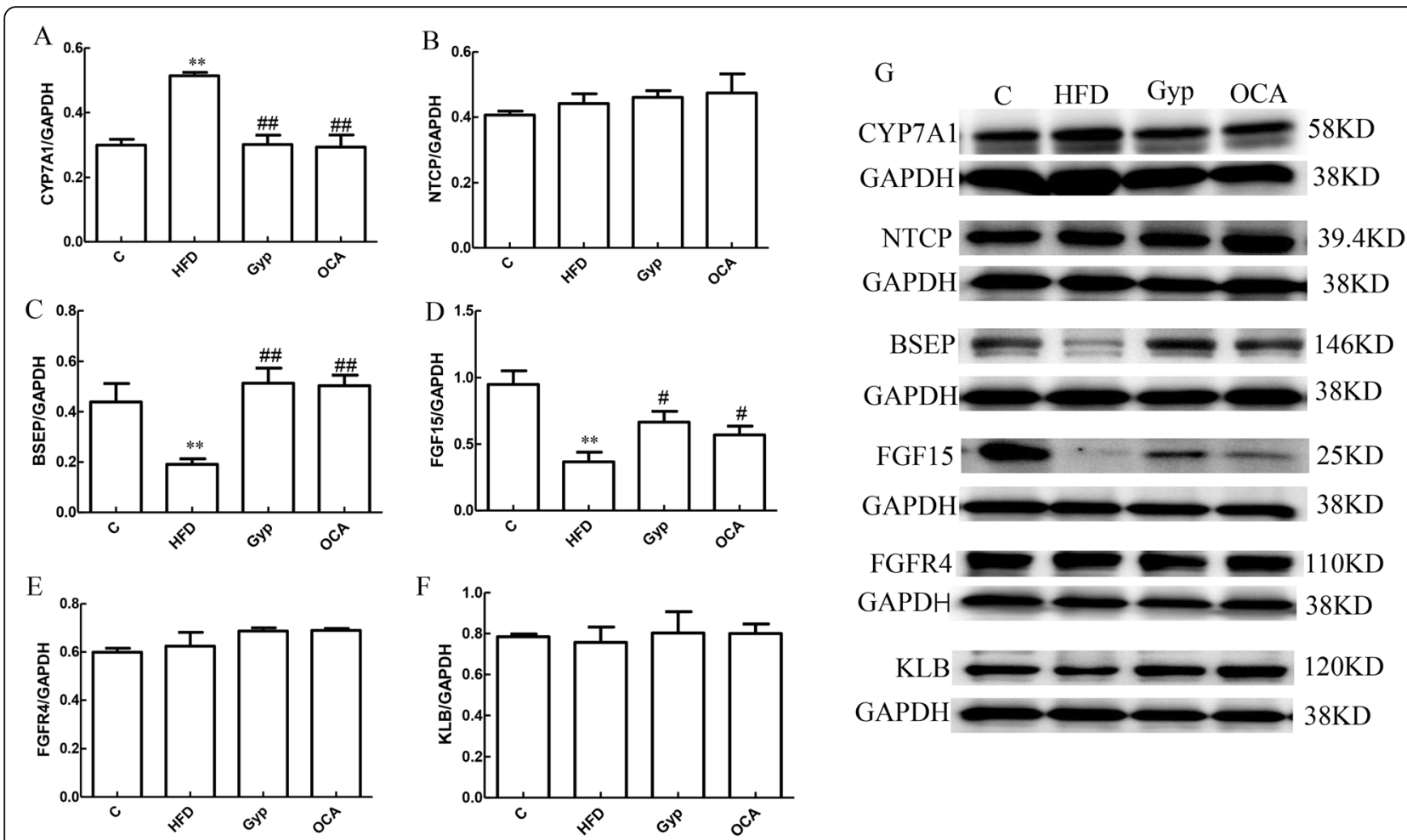

Fig. 11 Changes in FGF15, FGFR4, KLB, CYP7A1, NTCP, and BSEP protein content in liver tissues among the different experimental groups. a Bar chart representing the relative CYP7A1 protein expression level in liver tissue, $\mathbf{b}$ relative NTCP protein expression level, $\mathbf{c}$ relative BSEP protein expression level, $\mathbf{d}$ relative FGF15 protein expression level, e relative FGFR4 protein expression level, and $\mathbf{f}$ relative KLB protein expression level in liver tissue. g Representative autoradiograph image of western blotting with the different antibodies. ${ }^{* *} P<0.01$, vs control group; ${ }^{\# \#} P<0.01,{ }^{\#} P<$ 0.05 , vs HFD group. Data are presented as mean \pm standard deviation, $n=3$. C, control; HFD, high-fat diet; Gyp, gypenosides; OCA, obeticholic acid

\section{Discussion}

NAFLD is a chronic liver disorder that impacts approximately $24 \%$ of the adult population worldwide [28]. Steatosis or increased accumulation of intrahepatic TGs is a characterizing feature of NAFLD [29]. Bile acids are crucial for lipid metabolism, and they are exclusively synthesized in hepatocytes [30]. Bile acids are the end products of cholesterol metabolism [31]. Upon entering the intestine, primary bile acids promote lipid digestion and are converted to secondary bile acids by intestinal bacteria. Roughly $95 \%$ of intestinal bile acids are absorbed by the intestinal wall and then return to the liver via the portal vein. From the hepatocytes, the absorbed bile acids along with newly formed conjugated bile acids are discharged into the intestinal tract through the bile duct in a process named the enterohepatic circulation of bile acids [32]. Bile acid homeostasis is essential for maintaining normal lipid metabolism [33, 34]. Therefore, accumulating evidence suggests that abnormal bile acid metabolism can lead to the dysregulation of lipid metabolism, which can promote the occurrence and development of NAFLD [35-37]. Moreover, Lu et al. demonstrated that children with NAFLD had altered bile acid profiles and elevated serum TBA levels [38]. In NAFLD, bile acids can improve inflammation and glucolipid metabolism through the regulation of FXR and TGR5 expression and their downstream signaling pathways [35]. Therefore, bile acids and their receptors are crucial for maintaining normal systemic energy metabolism and hepatic lipid metabolism [39].

In this study, we induced NASH in mice by HFD feeding for 14 consecutive weeks. In agreement with previous reports [40], our results demonstrated the successful induction of NASH. We observed histopathological signs of hepatic steatosis along with increased liver TGs and NAS scores in the HFD group. Interestingly, treatment with Gyp or OCA (a FXR agonist) improved the histopathological picture and decreased the liver TG levels as well as the NAS scores. Moreover, we observed that Gyp treatment could significantly reduce the TBA content as well as the aMCA, TaMCA, BMCA, TßMCA TCDCA, CA, GCDCA, GCA and TCA content and significantly increased the alloLCA and $\beta C D C A$ content in liver tissues from NASH mice, and thus, it promoted hepatic bile acid homeostasis. In agreement, previous research demonstrated that Gyp can alter the liver bile acid 


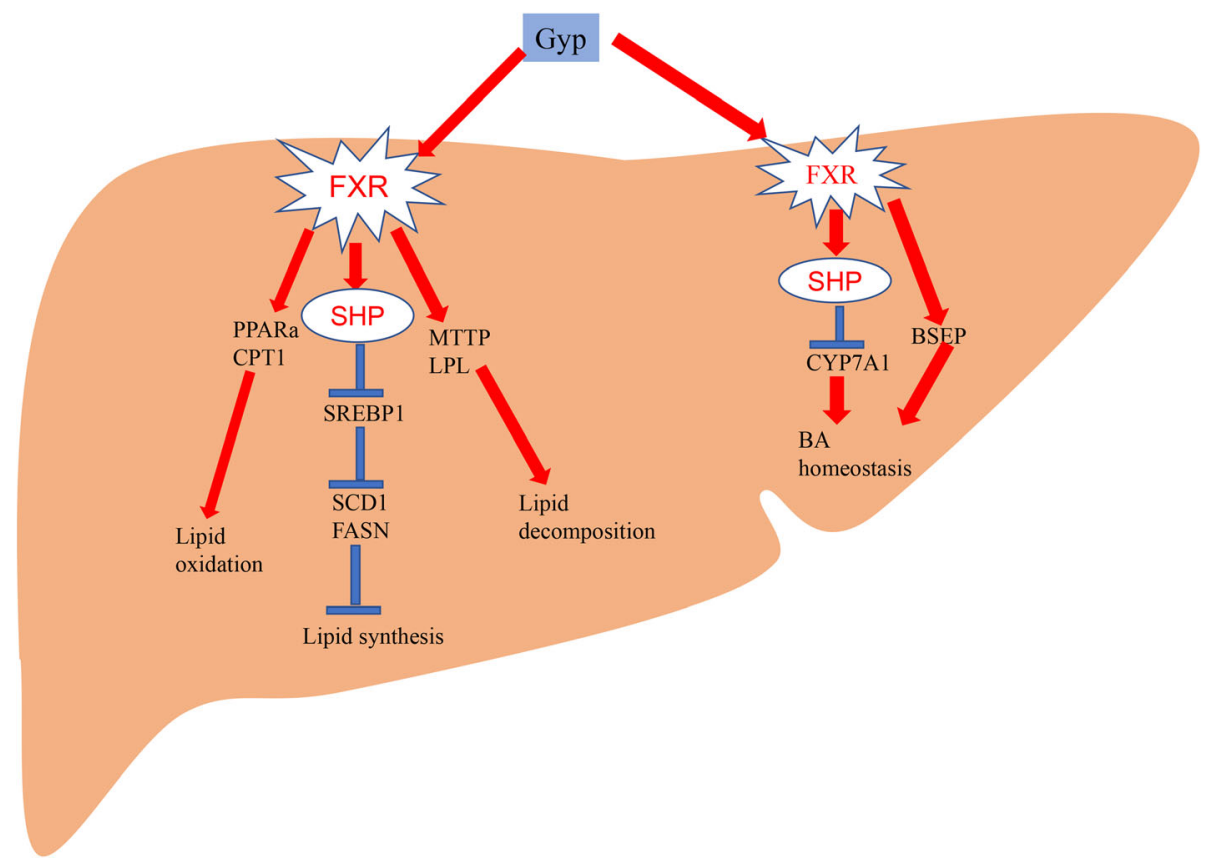

Fig. 12 Graphical abstract. Gypenosides treatment upregulates the SHP level and downregulates the SREBP1 level, thereby decreasing SCD1 and FASN expression and inhibiting hepatic lipid synthesis. Meanwhile, gypenosides can upregulate the expression of PPARa and CPT1 and enhance fatty acid oxidation, gypenosides can also upregulate LPL and MTTP expression, which led to the promotion of lipid decomposition. In addition, gypenosides treatment could significantly upregulate FXR-mediated bile acid pathways, thereby maintaining bile acid homeostasis. FXR, farnesoid $X$ receptor; SHP, small heterodimer partner; SREBP1, sterol-regulatory element-binding protein 1; SCD1, stearyl coenzyme A desaturation enzyme 1; FASN, fatty acid synthetase; PPARa, peroxisome proliferator-activated receptor alpha; CPT1, carnitine palmitoyl transferase 1; LPL, lipoprotein lipase; MTTP, microsomal triglyceride transfer protein. CYP7A1, cholesterol 7-alpha hydroxy-lase; BSEP, bile salt export protein

homeostasis induced by a HFD [41]. Also, Gyp treatment successfully regulated the adipose thermogenesis and gut microbiota, which were reduced in the process of gaining weight [42]. Moreover, Gyp was shown to treat type 2 diabetes and NAFLD in rats [43]. Altogether, the abovementioned data suggest that Gyp have a beneficial effect on NAFLD. Indeed, our results confirmed the beneficial effect of Gyp on NASH. Notably, the biological impact of Gyp treatment was comparable to that of treatment with the FXR agonist, OCA. Therefore, these results suggest that Gyp and FXR can share a common pathway for the treatment of NASH.

In the normal physiological conditions, FXR requires bile acid activation and mediates negative feedback regulation of bile acid metabolism [44, 45]. FXR is crucial for the regulation of bile acid synthesis, its transportation and maintenance of bile acid homeostasis [46]. Upon activation, FXR can increase the expression of SHP in the liver tissue, thereby inhibiting CYP7A1 expression, regulating NTCP and BSEP expression, and improving bile acid metabolism [47, 48]. Alternatively, FXR can upregulate the expression of FGF15 in the small intestine. The interaction of FGF15 and FGFR4 in the liver, along with KLB can ultimately inhibit the expression of CYP7A1, a key enzyme in bile acid synthesis [49]. Inhibition of the
FXR signaling pathway can lead to abnormal bile acid metabolism [50]. In this study, treatment with OCA upregulated the expression of FXR, SHP, FGF15 and BSEP in liver, which was in agreement with previously published results [48]. Furthermore, our results demonstrated that Gyp significantly upregulated the levels of FXR, SHP, FGF15 and BSEP and downregulated the level of CYP7A1 in liver tissues. Therefore, it is plausible to speculate that Gyp can improve bile acid metabolism and maintain bile acid homeostasis by regulating FXRmediated bile acids metabolic pathways.

FXR plays an important role in the regulation of lipid metabolism [51]. Upon activation, FXR can regulate lipid metabolism and maintain bile acid homeostasis primarily through the following pathways: 1) upregulation of SHP expression and inhibition of sterol-regulatory elementbinding protein 1c (SREBP-1c) expression [51, 52]. SREBP-1c is a key regulator of lipogenesis, and its downregulation can significantly decrease the expression of lipogenesis-related genes, like FASN and SCD1, thereby inhibiting lipid deposition [53]; 2) activation of PPAR $\alpha$, upregulation of CPT-1 expression, and promotion of fatty acid oxidation [52];3) induction of apolipoprotein CII (ApoCII) expression and inhibition of apolipoprotein CIII (ApoCIII) expression, thereby activating LPL, which 
promotes TG decomposition [54]; and 4) upregulation of MTTP and very low-density lipoprotein receptor (VLDLR) expression, thereby promoting the transportation and clearance of TG from the liver [55].

The results obtained from this study revealed that Gyp significantly increased the FXR mRNA and protein levels in liver tissues of mice with NASH. Similarly, Gyp treatment increased the SHP level and downregulated the SREBP1 mRNA expression and protein content in liver tissues, thereby decreasing the SCD1 and FASN expression and inhibiting hepatic lipid synthesis. In addition, Gyp treatment upregulated the expression of PPAR $\alpha$ and CPT1 in the liver tissues at both the mRNA and protein levels, thereby enhancing fatty acid oxidation. Further, Gyp upregulated LPL and MTTP expression, which led to the promotion of lipid decomposition. Therefore, Gyp are capable of regulating the lipid metabolic pathways by upregulating FXR expression, which can ultimately lead to an improvement in lipid metabolism in NAFLD.

\section{Conclusions}

In conclusion, we confirmed that Gyp can significantly reduce the lipid content in the liver and serum, reduce serum ALT and AST activities, improve insulin resistance and ameliorate pathological changes in liver tissues. These results suggest the beneficial impact of Gyp in the treatment of NAFLD. In addition, Gyp treatment could significantly upregulate FXR-mediated bile acid and lipid metabolism pathways, thereby maintaining bile acid and lipid homeostasis. Interestingly, the impact of Gyp on the downstream bile acid and lipid signaling molecules was similar to that of FXR agonist treatment (OCA). Therefore, it is possible to hypothesize that the function of Gyp maybe related to activation of the FXR signaling pathway (Fig. 12).

\footnotetext{
Abbreviations

Gyp: Gypenosides; FXR: Farnesoid X receptor; SHP: Small heterodimer partner; SREBP1: Sterol-regulatory element-binding protein 1; SCD1: Stearyl coenzyme A desaturation enzyme 1; FASN: Fatty acid synthetase; PPARa: Peroxisome proliferator-activated receptor alpha; CPT1: Carnitine palmitoyl transferase 1; MTTP: Microsomal triglyceride transfer protein; CYP7A1: Cholesterol 7-alpha hydroxy-lase; FGFR4: Fibroblast growth factor receptor 4; BSEP: Bile salt export protein; NTCP: Na+-taurocholate cotransporting polypeptide; KLB: Klotho beta; FGF15: Fibroblast growth factor 15; LPL: Lipoprotein lipase; HFD: High-fat diet; OCA: Obeticholic acid; ALT: Alanine aminotransferase; AST: Aspartate aminotransferase; FINS: Fasting insulin; FBG: Fasting blood glucose; HOMA-IR: Homeostatic Model Assessment for Insulin Resistance; TG: Triglycerides; TC: Total cholesterol; LDLC: Low density lipoprotein cholesterol; HDL-C: High density lipoprotein cholesterol; TBA: Total bile acid; aMCA: a-muricholic acid; $\beta$ MCA: $\beta$ muricholic acid; TaMCA: Tauro a-muricholic acid; T $\beta M C A$ : Tauro $\beta$-muricholic acid; alloLCA: allolithocholic acid; TCDCA: Taurochenodeoxycholic acid; CA: Cholic acid; $\beta C D C A$ : $3 \beta$-chenodeoxycholic acid; GCDCA: Glycochenodeoxycholic acid; TCA: Taurocholic acid; GCA: Glycocholic acid
}

\section{Acknowledgments}

None

\section{Authors' contributions}

HSL and YYH conceived and designed research; YFX, XX and HJT collected data and conducted research; HSL, XX and HJT analyzed and interpreted data; HSL and YFX wrote the initial paper; YYH revised the paper; HSL and YYH had primary responsibility for final content. All authors read and approved the final manuscript.

\section{Funding}

This study was supported by grants-in-aid from the National Natural Science Foundation of China (81873109).

\section{Availability of data and materials}

The datasets generated and analyzed during the current study are available from the corresponding author on reasonable request.

\section{Ethics approval and consent to participate}

The experimental protocol was revised and approved by the Experimental Animal Ethics Committee of Ningbo University. All procedures performed in studies involving animals were in accordance with the ethical standards of the institution or practice at which the studies were conducted.

\section{Consent for publication}

All data published here are under the consent for publication.

\section{Competing interests}

The authors declare that they have no competing interests.

\section{Author details}

${ }^{1}$ Institute of Liver Disease, Shuguang Hospital, Shanghai University of Traditional Chinese Medicine, Shanghai, China. 'Liver Disease Department, Hwa Mei Hospital, University of Chinese Academy of Sciences, Ningbo, Zhejiang, China. ${ }^{3}$ Key Laboratory of Diagnosis and Treatment of Digestive System Tumors of Zhejiang Province, Ningbo 315010, Zhejiang, China.

${ }^{4}$ Medical School of Ningbo University, Ningbo, Zhejiang, China.

Received: 21 November 2019 Accepted: 22 April 2020

Published online: 01 May 2020

\section{References}

1. Rinella ME. Nonalcoholic fatty liver disease: a systematic review. JAMA. 2015; 313:2263-73.

2. Jiao N, Baker SS, Chapa-Rodriguez A, Liu W, Nugent CA, Tsompana M, et al. Suppressed hepatic bile acid signalling despite elevated production of primary and secondary bile acids in NAFLD. Gut. 2018:67:1881-91.

3. Brunt EM. Histopathology of non-alcoholic fatty liver disease. Clin Liver Dis. 2009;13:533-44.

4. Margini C, Dufour JF. The story of HCC in NAFLD: from epidemiology, across pathogenesis, to prevention and treatment. Liver Int. 2016;36:317-24.

5. Younossi Z, Anstee QM, Marietti M, Hardy T, Henry L, Eslam M, et al. Global burden of NAFLD and NASH: trends, predictions, risk factors and prevention. Nat Rev Gastroenterol Hepatol. 2018;15:11-20.

6. Hung CK, Bodenheimer HC Jr. Current treatment of nonalcoholic fatty liver disease/nonalcoholic Steatohepatitis. Clin Liver Dis. 2018;22:175-87.

7. Ji X, Shen Y, Guo X. Isolation, Structures, and Bioactivities of the Polysaccharides from Gynostemma pentaphyllum (Thunb.) Makino: A Review. Biomed Res Int. 2018;2018:6285134.

8. Hong M, Cai Z, Song L, Liu Y, Wang Q, Feng X. Gynostemma pentaphyllum attenuates the progression of nonalcoholic fatty liver disease in mice: a biomedical investigation integrated with in Silico assay. Evid Based Complement Alternat Med. 2018;2018:8384631.

9. Liu J, Zhang L, Ren Y, Gao Y, Kang L, Qiao Q. Anticancer and immunoregulatory activity of Gynostemma pentaphyllum polysaccharides in H22 tumor-bearing mice. Int J Biol Macromol. 2014;69:1-4.

10. Jia N, Lin X, Ma S, Ge S, Mu S, Yang C, et al. Amelioration of hepatic steatosis is associated with modulation of gut microbiota and suppression of hepatic miR-34a in Gynostemma pentaphylla (Thunb.) Makino treated mice. Nutr Metab (Lond). 2018;15:86.

11. Shen CY, Jiang JG, Shi MM, Yang HL, Wei H, Zhu W. Comparison of the effects and inhibitory pathways of the constituents from Gynostemma pentaphyllum against LPS-induced inflammatory response. J Agric Food Chem. 2018;66:11337-46. 
12. Wang J, Yang JL, Zhou PP, Meng XH, Shi YP. Further new Gypenosides from Jiaogulan (Gynostemma pentaphyllum). J Agric Food Chem. 2017;65:592634.

13. Aktan F, Henness S, Roufogalis BD, Ammit AJ. Gypenosides derived from Gynostemma pentaphyllum suppress NO synthesis in murine macrophages by inhibiting iNOS enzymatic activity and attenuating NF-kappaB-mediated iNOS protein expression. Nitric Oxide. 2003;8:235-42.

14. Li H, Ying H, Hu A, Hu Y, Li D. Therapeutic effect of Gypenosides on nonalcoholic Steatohepatitis via regulating hepatic Lipogenesis and fatty acid oxidation. Biol Pharm Bull. 2017;40:650-7.

15. Kim SG, Kim BK, Kim K, Fang S. Bile acid nuclear receptor Farnesoid X receptor: therapeutic target for nonalcoholic fatty liver disease. Endocrinol Metab (Seoul). 2016:31:500-4

16. Neuschwander-Tetri BA, Loomba R, Sanyal AJ, Lavine JE, Van Natta ML, Abdelmalek MF, et al. Farnesoid X nuclear receptor ligand obeticholic acid for non-cirrhotic, non-alcoholic steatohepatitis (FLINT): a multicentre, randomised, placebo-controlled trial. Lancet. 2015;385:956-65.

17. Dong R, Yang X, Wang C, Liu K, Liu Z, Ma X, et al. Yangonin protects against non-alcoholic fatty liver disease through farnesoid $X$ receptor. Phytomedicine. 2019;53:134-42.

18. Cipriani S, Mencarelli A, Palladino G, Fiorucci S. FXR activation reverses insulin resistance and lipid abnormalities and protects against liver steatosis in Zucker (fa/fa) obese rats. J Lipid Res. 2010;51:771-84.

19. Zheng Z, Zhao Z, Li S, Lu X, Jiang M, Lin J, et al. Altenusin, a nonsteroidal microbial metabolite, attenuates nonalcoholic fatty liver disease by activating the Farnesoid X receptor. Mol Pharmacol. 2017;92:425-36.

20. Li XM, Peng JH, Sun ZL, Tian HJ, Duan XH, Liu L, et al. Chinese medicine CGA formula ameliorates DMN-induced liver fibrosis in rats via inhibiting MMP2/9, TIMP1/2 and the TGF-beta/Smad signaling pathways. Acta Pharmacol Sin. 2016:37:783-93.

21. Qu H, Wang Y, Wang Y, Yang T, Feng Z, Qu Y, et al. Luhong formula inhibits myocardial fibrosis in a paracrine manner by activating the gp130/JAK2/ STAT3 pathway in cardiomyocytes. J Ethnopharmacol. 2017;202:28-37.

22. Massafra V, Milona A, Vos HR, Ramos RJJ, Gerrits J, Willemsen ECL, et al. Farnesoid $X$ receptor activation promotes hepatic amino acid catabolism and ammonium clearance in mice. Gastroenterology. 2017;152:1462-76 e10.

23. Li H, Ying H, Hu A, Li D, Hu Y. Salidroside modulates insulin signaling in a rat model of nonalcoholic Steatohepatitis. Evid Based Complement Alternat Med. 2017;2017:9651371

24. Kleiner DE, Brunt EM, Van Natta M, Behling C, Contos MJ, Cummings OW, et al. Design and validation of a histological scoring system for nonalcoholic fatty liver disease. Hepatology. 2005;41:1313-21.

25. Fraulob JC, Ogg-Diamantino R, Fernandes-Santos C, Aguila MB, Mandarimde-Lacerda CA. A mouse model of metabolic syndrome: insulin resistance, fatty liver and non-alcoholic fatty pancreas disease (NAFPD) in C57BL/6 mice fed a high fat diet. J Clin Biochem Nutr. 2010;46:212-23.

26. Xie G, Wang X, Huang F, Zhao A, Chen W, Yan J, et al. Dysregulated hepatic bile acids collaboratively promote liver carcinogenesis. Int J Cancer. 2016; 139:1764-75.

27. Lan K, Su M, Xie G, Ferslew BC, Brouwer KL, Rajani C, et al. Key role for the 12-Hydroxy Group in the Negative ion Fragmentation of unconjugated C24 bile acids. Anal Chem. 2016;88:7041-8.

28. Araujo AR, Rosso N, Bedogni G, Tiribelli C, Bellentani S. Global epidemiology of non-alcoholic fatty liver disease/non-alcoholic steatohepatitis: what we need in the future. Liver Int. 2018;38(Suppl 1):47-51.

29. Fabbrini E, Sullivan S, Klein S. Obesity and nonalcoholic fatty liver disease: biochemical, metabolic, and clinical implications. Hepatology. 2010;51:679-89.

30. Staels B, Fonseca VA. Bile acids and metabolic regulation: mechanisms and clinical responses to bile acid sequestration. Diabetes Care. 2009;32(Suppl 2):S237-45.

31. Yu Q, Jiang Z, Zhang L. Bile acid regulation: a novel therapeutic strategy in non-alcoholic fatty liver disease. Pharmacol Ther. 2018;190:81-90.

32. Dawson PA. Hepatic bile acid uptake in humans and mice: multiple pathways and expanding potential role for gut-liver signaling. Hepatology. 2017:66:1384-6.

33. Sun R, Yang N, Kong B, Cao B, Feng D, Yu X, et al. Orally administered Berberine modulates hepatic lipid metabolism by altering microbial bile acid metabolism and the intestinal FXR signaling pathway. Mol Pharmacol. 2017:91:110-22.

34. Do HJ, Lee YS, Ha MJ, Cho Y, Yi H, Hwang YJ, et al. Beneficial effects of voglibose administration on body weight and lipid metabolism via gastrointestinal bile acid modification. Endocr J. 2016;63:691-702.
35. Chavez-Talavera O, Tailleux A, Lefebvre P, Staels B. Bile acid control of metabolism and inflammation in obesity, type 2 diabetes, dyslipidemia, and nonalcoholic fatty liver disease. Gastroenterology. 2017;152:1679-94 e3.

36. Li Y, Jadhav $K$, Zhang Y. Bile acid receptors in non-alcoholic fatty liver disease. Biochem Pharmacol. 2013;86:1517-24.

37. Yuan L, Bambha K. Bile acid receptors and nonalcoholic fatty liver disease. World J Hepatol. 2015;7:2811-8.

38. Lu LP, Wan YP, Xun PC, Zhou K, Chen C, Cheng SY, et al. Serum bile acid level and fatty acid composition in Chinese children with non-alcoholic fatty liver disease. J Dig Dis. 2017;18:461-71.

39. Rao A, Kosters A, Mells JE, Zhang W, Setchell KD, Amanso AM, et al. Inhibition of ileal bile acid uptake protects against nonalcoholic fatty liver disease in high-fat diet-fed mice. Sci Transl Med. 2016;8:357ra122.

40. Peng JH, Leng J, Tian HJ, Yang T, Fang Y, Feng Q, et al. Geniposide and Chlorogenic acid combination ameliorates non-alcoholic Steatohepatitis involving the protection on the gut barrier function in mouse induced by high-fat diet. Front Pharmacol. 2018;9:1399.

41. Lu Y, Du Y, Qin L, Wu D, Wang W, Ling L, et al. Gypenosides altered hepatic bile acids homeostasis in mice treated with high fat diet. Evid Based Complement Alternat Med. 2018;2018:8098059.

42. Liu J, Li Y, Yang P, Wan J, Chang Q, Wang TTY, et al. Gypenosides reduced the risk of overweight and insulin resistance in C57BL/6J mice through modulating adipose thermogenesis and gut microbiota. J Agric Food Chem. 2017:65:9237-46.

43. He Q, Li JK, Li F, Li RG, Zhan GQ, Li G, et al. Mechanism of action of gypenosides on type 2 diabetes and non-alcoholic fatty liver disease in rats. World J Gastroenterol. 2015;21:2058-66.

44. Kong B, Wang L, Chiang JY, Zhang Y, Klaassen CD, Guo GL. Mechanism of tissue-specific farnesoid $X$ receptor in suppressing the expression of genes in bile-acid synthesis in mice. Hepatology. 2012;56:1034-43.

45. Li G, Guo LG. Farnesoid X receptor, the bile acid sensing nuclear receptor, in liver regeneration. Acta Pharm Sin B. 2015;5:93-8.

46. Pathak P, Xie C, Nichols RG, Ferrell JM, Boehme S, Krausz KW, et al. Intestine farnesoid $X$ receptor agonist and the gut microbiota activate $G$-protein bile acid receptor-1 signaling to improve metabolism. Hepatology. 2018;68: 1574-88.

47. Xiang D, Yang J, Liu Y, He W, Zhang S, Li X, et al. Calculus Bovis Sativus improves bile acid homeostasis via Farnesoid $X$ receptor-mediated signaling in rats with estrogen-induced cholestasis. Front Pharmacol. 2019;10:48.

48. Zhang Y, Jackson JP, St Claire RL 3rd, Freeman K, Brouwer KR, Edwards JE. Obeticholic acid, a selective farnesoid $X$ receptor agonist, regulates bile acid homeostasis in sandwich-cultured human hepatocytes. Pharmacol Res Perspect. 2017:5:e00329.

49. Hartmann $P$, Hochrath $K$, Horvath $A$, Chen $P$, Seebauer $C T$, Llorente $C$, et al. Modulation of the intestinal bile acid/farnesoid $X$ receptor/fibroblast growth factor 15 axis improves alcoholic liver disease in mice. Hepatology. 2018;67: 2150-66.

50. Kong B, Zhang M, Huang M, Rizzolo D, Armstrong LE, Schumacher JD, et al. FXR deficiency alters bile acid pool composition and exacerbates chronic alcohol induced liver injury. Dig Liver Dis. 2019;51:570-6.

51. Arab JP, Karpen SJ, Dawson PA, Arrese M, Trauner M. Bile acids and nonalcoholic fatty liver disease: molecular insights and therapeutic perspectives. Hepatology. 2017;65:350-62.

52. Duan X, Meng Q, Wang C, Liu Z, Liu Q, Sun H, et al. Calycosin attenuates triglyceride accumulation and hepatic fibrosis in murine model of nonalcoholic steatohepatitis via activating farnesoid $X$ receptor. Phytomedicine. 2017;25:83-92.

53. Zhang Y, Meng T, Zuo L, Bei Y, Zhang Q, Su Z, et al. Xyloketal B attenuates fatty acid-induced lipid accumulation via the SREBP-1c pathway in NAFLD models. Mar Drugs. 2017;15:163.

54. Mazuy C, Helleboid A, Staels B, Lefebvre P. Nuclear bile acid signaling through the farnesoid X receptor. Cell Mol Life Sci. 2015;72:1631-50.

55. Fuchs CD, Traussnigg SA, Trauner M. Nuclear receptor modulation for the treatment of nonalcoholic fatty liver disease. Semin Liver Dis. 2016:36:69-86.

\section{Publisher's Note}

Springer Nature remains neutral with regard to jurisdictional claims in published maps and institutional affiliations. 